\title{
Effect of surrogate design on the measured stiffness of snowboarding wrist protectors
}

\author{
Caroline Adams ${ }^{1}$ (D) David James $^{1}$ (D) Terry Senior $^{1}$ (I) $\cdot$ Tom Allen $^{2}$ (I) $\cdot$ Nick Hamilton $^{1}$ (I)
}

Published online: 7 March 2018

(C) The Author(s) 2018. This article is an open access publication

\begin{abstract}
In snowboarding, the wrist is the most common injury site, as snowboarders often put their arms out to cushion a fall. This can result in a compressive load through the carpals coupled with wrist hyperextension, leading to ligament sprains or carpal and forearm bone fractures. Wrist protectors are worn by snowboarders in an effort to reduce injury risk, by decreasing peak impact forces and limiting wrist extension to prevent hyperextension during falls. There is no international standard or universally accepted performance specification that snowboarding wrist protectors should conform to, resulting in an inability to judge which designs offer the best protection. This study investigated how surrogate arm design affected the stiffness of wrist protectors during quasi-static mechanical testing. Three surrogate arms with increasing design complexity were used to test three wrist protectors. The results show that surrogate design does influence the stiffness of snowboarding wrist protectors. Given that the surrogate does influence protector performance, it is recommended that a standard surrogate design is established for research and product testing.
\end{abstract}

Keywords Injury prevention $\cdot$ Mechanical testing $\cdot$ Protective equipment $\cdot$ Snowboarding falls

\section{Introduction}

There are an estimated 10-15 million snowboarders worldwide [1]. The risk of sustaining an injury while snowboarding is higher than alpine skiing [2-4] and injury rates are among the highest of all sports in the 9 to 19-yearold age group [5]. In snowboarding, the wrist is the most frequently injured region [6-8], with wrist fractures a common occurrence [9]. Snowboarders often attempt to cushion a fall with outstretched hands. In this scenario impact loads can be transmitted along the upper extremity as an axial compression force and extension torque resulting in wrist hyperextension, which can lead to ligament sprains or carpal and forearm bone fractures $[10,11]$.

Different preventative measures can be adopted: changing the biomechanical response of the body; altering

Caroline Adams

caroline.adams@shu.ac.uk

1 Centre for Sports Engineering Research, Sheffield Hallam University, Sheffield, UK

2 School of Engineering, Manchester Metropolitan University, Manchester, UK how the applied load is distributed and reducing injury risk through the application of engineering design and appropriate regulation [12], including (1) the design of ski areas, such as terrain park jumps $[13,14]$ and (2) personal protective equipment (PPE) such as helmets [15]. Wrist protectors have been adopted amongst snowboarders as a preventative measure to: (1) limit peak impact forces, (2) absorb or shunt the impact energy, and (3) prevent hyperextension $[1,16]$. A range of wrist protectors of differing designs are available. A common approach is to include features intended to stiffen the wrist to prevent hyperextension, such as splints on the palmar and dorsal sides of the wrist $[1,17]$. There is no universal specification for assessing snowboarding wrist protector performance, but following a call in 2013 [1], the ISO/CD 20320 was setup to develop a standard for these products [18]. The European standard EN 14120:2003 prescribes requirements for roller sports wrist protectors and has been identified as a suitable starting point for developing a dedicated snowboarding wrist protector standard [17]. One of the requirements of EN 14120 is that protectors undergo a test to measure their stiffness (EN 14120 requirement 5.9) 
which is thought to relate to their ability to prevent wrist hyperextension.

Adams et al. [19] critiqued EN 14120 stiffness test protocol and presented a modified test to assess the stiffness of snowboarding wrist protectors. Where stiffness is defined as the protector's ability to limit wrist extension under load. A bespoke rig fitted to a uniaxial testing device was used to facilitate testing of protectors over a range of torques rather than just one $(3 \mathrm{Nm})$ as prescribed in $\mathrm{EN}$ 14120. The surrogate prescribed in EN 14120 was used, but found to be unsuitable for testing all designs of snowboarding wrist protectors as a lack of fingers did not allow assessment of products integrated into gloves. In addition, there is no evidence to suggest that the simplified design (shape and size) of the surrogate was based on anthropometric data. Given the need for a new surrogate, the aim of the current study was to investigate the influence of surrogate design (both shape and size) on the measured stiffness of snowboarding wrist protectors. Two new surrogates of increasing complexity were developed and the stiffness of three protectors was compared for the two new designs and the EN 14120 surrogate. For the purpose of this study, a well-designed surrogate is considered to be based on anthropometric dimensions, to enable testing of both stand-alone protectors and those integrated into gloves, and detect differences between products in a repeatable manner. A better understanding of wrist protector performance on different surrogates will assist in the design of surrogates for the proposed snowboard specific International Standard ISO/CD 20320.

\section{Method}

Two new surrogate arms of increasing biofidelity were developed (Fig. 1) and used to test the stiffness of three wrist protectors. The new surrogates were compared with the surrogate outlined in EN 14120 roller sports protective equipment standard (Fig. 1a), that was used by Adams et al. [19]. All three surrogates correspond approximately to EN 420 sizes 8 and 9; a summary of their measurements is given in Table 1. The EN 14120 surrogate has a simple shape with a uniform rectangular cross-section forearm and a paddle-like hand with no fingers.

The first new surrogate (geometric, Fig. 1b) is more biofidelic in terms of shape and size. It is a simplified geometric representation of a hand-arm designed using computer-aided design (CAD) software (Pro/ENGINEER, USA) based on eleven dimensions from anthropometric datasets, equivalent to European hand size 8 [20-23]. Simplifications of the hand form resulted in a scalable design constructed from simple geometric profiles that can be communicated in an engineering drawing and reproduced globally. This surrogate is the proposed size medium surrogate in the draft version of ISO/CD 20320 standard (E update 2017-03-22) for snowboarding wrist protectors [18].

The second new surrogate (scanned, Fig. 1c) was the most biofidelic of the three and was created from a 3D scan (3dMD, USA) of a human hand and arm. A participant with hand measurements close to published 50th percentile data (equivalent to hand size 8/9) was identified from a sample of ten, based on nine manual measurements of each upper extremity: forearm circumference, wrist circumference over bony protrusion and at wrist crease, wrist width, hand circumference, hand breadth, hand length, middle finger length and palm length. The difference between each participant and published 50th percentile data [21] was determined for each measure, the summed squared error across all measures was then calculated and the participant with the lowest value deemed the most appropriate forearm to base the surrogate on. The selected participant had a sum squared error of $917 \mathrm{~mm}$ and all measurements were within $10 \%$ of the 50 th percentile, the largest differences from the 50th percentile measurements were the wrist and forearm circumferences. Ethical approval was obtained from the Faculty of Health and Wellbeing Ethics Committee, Sheffield Hallam University, UK (HWB-S\&E-69). The scan data point cloud was manipulated and converted to a surface before adding a wrist hinge joint using CAD software (Geomagic, USA and Pro/ENGINEER, USA).

The three surrogates will be referred to as EN 14120, geometric and scanned. All three surrogate arms and the two new surrogates hands were made from solid polyamide (tensile modulus $1650 \mathrm{MPa} \pm 150$ [24]) using laser sintering (Materialise, UK). The EN 14120 surrogate hand was made from polyamide (tensile modulus $3309 \mathrm{MPa}$ [25]) using fused deposition modelling (Makerbot, USA). A single axis low friction hinge joint was used to mimic the flexion extension motion of the wrist as stipulated in EN 14120. To facilitate testing of products integrated into gloves, two steel rods $(\varnothing 12 \times 80 \mathrm{~mm})$ imitating digits three and four were incorporated into the geometric and scanned surrogate and a clamp was used to attach the wire which transferred displacement from the testing device (Fig. 2). To ensure protectors could be mounted onto the surrogates, they each included a protrusion in place of a full thumb.

Three different commercially available adult wrist protectors were tested (Fig. 3). Two snowboarding protectors were chosen, as they represent different design approaches, whilst the roller sports protector acted as a comparison that was certified to EN 14120 (Table 2). Based on protector dimensions, the two snowboarding protectors will be referred to here as short and long snowboarding protector. Both snowboarding protectors were size medium, whilst 
Fig. 1 Surrogate designs that were compared a EN 14120, b geometric, c scanned. Dimension 1 is hand length from the centre of the hinge to the finger tips and dimension 2 is hand circumference over the knuckles

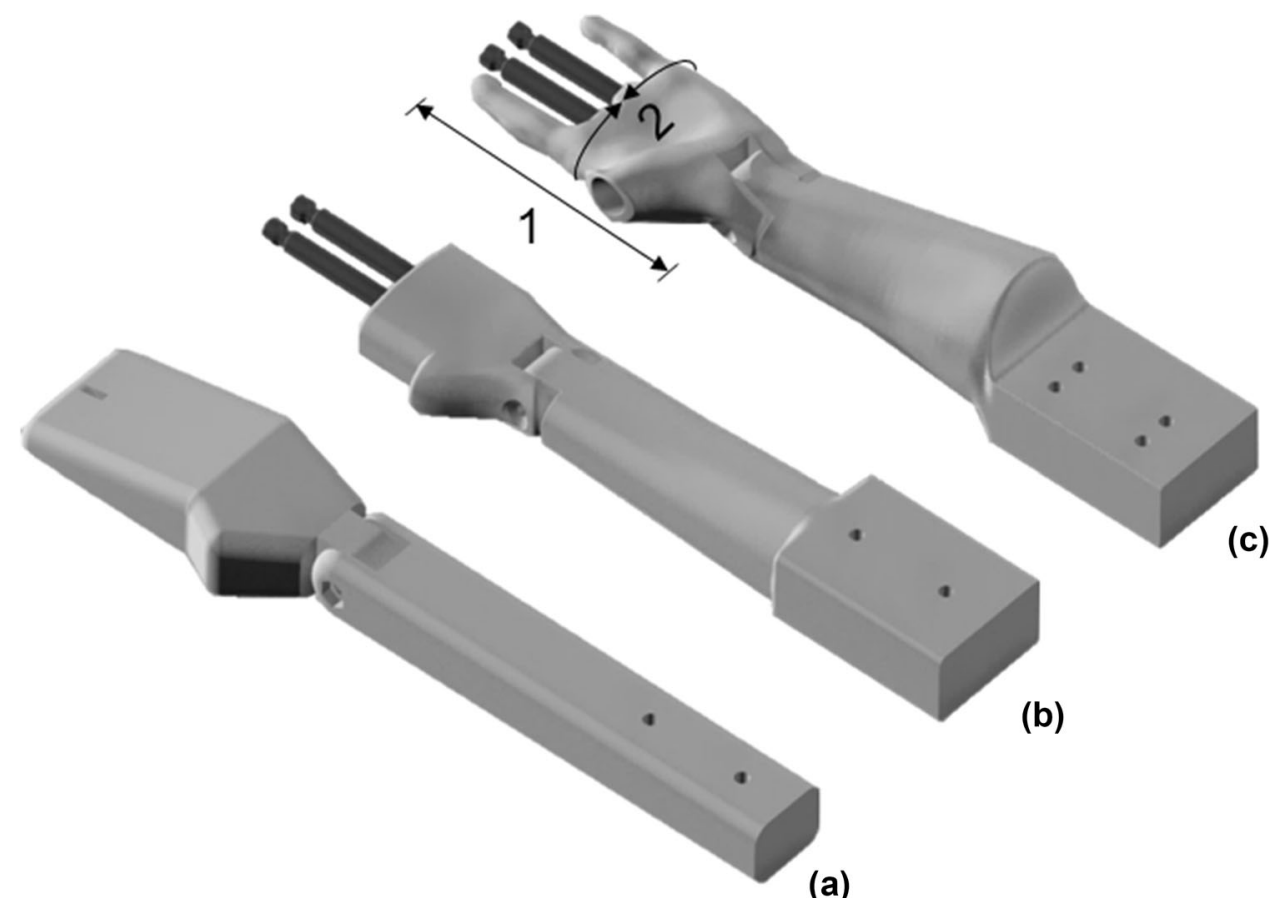

(c)

Table 1 Summary of surrogate measurements in relation to standard sizes

\begin{tabular}{llllll}
\hline Measurements & $\begin{array}{l}\text { 50th percentile male } \\
{[21]}\end{array}$ & $\begin{array}{l}\text { Size 8/9 measurements (EN } \\
420)\end{array}$ & $\begin{array}{l}\text { EN 14120 } \\
\text { surrogate }\end{array}$ & $\begin{array}{l}\text { Geometric } \\
\text { surrogate }\end{array}$ & $\begin{array}{l}\text { Scanned } \\
\text { surrogate }\end{array}$ \\
\hline Hand length (mm) & 190 & $182 / 192$ & 191 & 182 & 192 \\
Hand circumference $(\mathrm{mm})$ & 223 & $203 / 229$ & 220 & 200 & 207 \\
$\begin{array}{l}\text { Maximum forearm } \\
\quad \text { circumference }(\mathrm{mm})\end{array}$ & - & - & 167 & 197 & 240 \\
Total volume $\left(\mathrm{mm}^{3}\right)$ & - & - & 893,970 & 900,212 & $1,110,321$ \\
\hline
\end{tabular}

the roller sports protector was size large. Sizes were selected based on what fitted the EN 14120 surrogate, as there is no standard sizing used across manufacturers. Each protector was strapped by holding the surrogate horizontally, attaching a $2 \mathrm{~kg}$ mass to the strap and then rotating the surrogate about its long axis until the protector was securely fitted.

The experimental procedure was based on the approach outlined by Adams et al. [19] and is, therefore, only briefly summarised here. The surrogate was mounted to a bespoke rig and connected to a uniaxial testing machine (Instron 3367 , fitted with a $500 \mathrm{~N}$ load cell). Vertical displacement of the load cell at $200 \mathrm{~mm} / \mathrm{min}$ applied an extension torque to the wrist joint-via a cable attached to the fingers passing through a pulley-until the hand extension angle reached $\sim 90^{\circ}$ (Fig. 2). A preload of $\sim 1.5 \mathrm{~N}$ pre-tensioned the cable, removing any "slack" before the start of the trial. Load and displacement were recorded at $10 \mathrm{~Hz}$, with the start and end angle of the hand measured with an inclinometer (MW570-01, Moore \& Wright). Hand extension angle throughout the trial was inferred from the start and end angle, as load cell displacement rate was constant. Eight repeat trials were performed on each protector on each surrogate, resulting in 72 trials for the nine test conditions. The protector was re-positioned and restrapped between trials.

The load applied to the cable was measured in the vertical axis, rather than perpendicular to the hand as required to obtain the torque acting about the wrist joint of the surrogate. It was, therefore, necessary to calculate the load perpendicular to the hand throughout the trial. This perpendicular load was calculated from load cell data, start and end angle measurements and manual measurements of distances A, B, C and D (Fig. 2) using trigonometry as detailed in Adams et al. [19]. The relationship between hand angle and torque was studied for four cases: $35^{\circ}, 55^{\circ}$, $80^{\circ}$ and $90^{\circ}$. Angles $35^{\circ}$ and $55^{\circ}$ are the pass threshold in EN 14120 when $3 \mathrm{Nm}$ is applied, whilst $80^{\circ}$ and $90^{\circ}$ are 
Fig. 2 Schematic of test setup at start of test (solid line), part way (dashed line) and end of test (dot-dash line). 1 Load cell, 2 low friction pulley, 3 cable, 4 cable clamp, 5 surrogate steel fingers, 6 low friction joint, $\delta$ hand extension angle

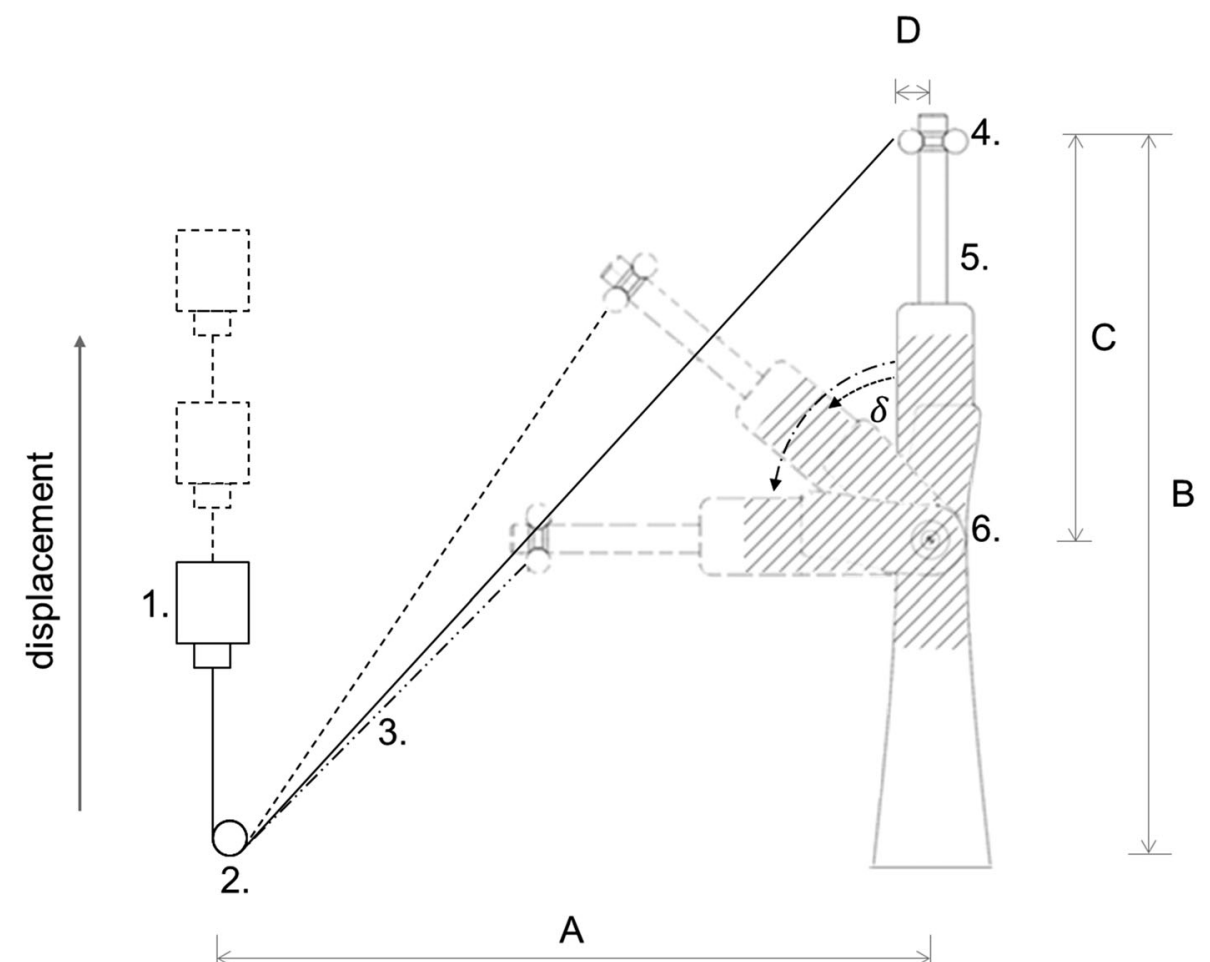

protector region

$A, B, C, D$ : manual measurements used to determine hand extension angle representative of wrist hyperextension and are comparable with wrist angles that have been measured in non-injurious on-slope falls [26]. Due to the sample frequency of the load cell $(10 \mathrm{~Hz})$, it was necessary to interpolate the results to define the torque at the prescribed angles, by fitting a firstorder polynomial function through the local data points (range $\leq 5^{\circ}$ ). Each of the eight repeats were analysed with the same method and a mean and standard deviation obtained. For statistical comparison, the data were split into thirty-six sets (3 surrogates $\times 3$ protectors $\times 4$ angles). Data was further divided into twelve groups to enable comparisons between surrogates to be made at each angle (e.g., same protector, same angle on three different surrogates).

The data was analysed with SPSS statistical software for analysis (IBM SPSS Statistics for Windows, USA). To test for normality and homogeneity of variance, Shapiro-Wilk and Levene tests were performed with the significance level set at $p<0.05$. Based on these outputs, statistical analysis tests were used for each of the twelve groups to determine differences in torque between the three surrogates at the same extension angle. Post hoc analyses were conducted to assess where the significant differences between pairs of surrogates occurred. One way ANOVA and Bonferroni post hoc were used if data were normally distributed and had equal variance; Welch ANOVA and Games Howell post hoc if data were normally distributed and had un-equal variance; Kruskal-Wallis and MannWhitney tests with a Bonferroni correction (effects reported at a 0.0167 level of significance) were used as a nonparametric equivalent to one way ANOVA. Effect sizes were calculated using Pearson's correlation coefficient, as described by Field [27]. The magnitudes of the correlations were interpreted using Cohen's thresholds where: $<0.1$, is trivial; $0.1-0.3$ is small; $0.3-0.5$ is moderate; and $>0.5$ is large [28]. To compare the repeatability of the three surrogates, the coefficient of variation was determined for each protector on each surrogate at the four angles of interest.

It was not possible to obtain full measurement sets for all four angles in two cases: $35^{\circ}$ for the roller sports protector mounted on the geometric surrogate and $90^{\circ}$ for the long snowboard protector mounted to the scanned surrogate. When the roller sports protector was mounted on the geometric surrogate, the start angle of the hand exceeded $35^{\circ}\left(47.5 \pm 2.2^{\circ}\right)$. Similar behaviour was observed in an earlier study; different protectors designs hold the hand at a different neutral angle [19]. The long snowboard protector 
Fig. 3 Three wrist protector models tested mounted onto geometric surrogate a short snowboarding, b long snowboarding, c roller sports

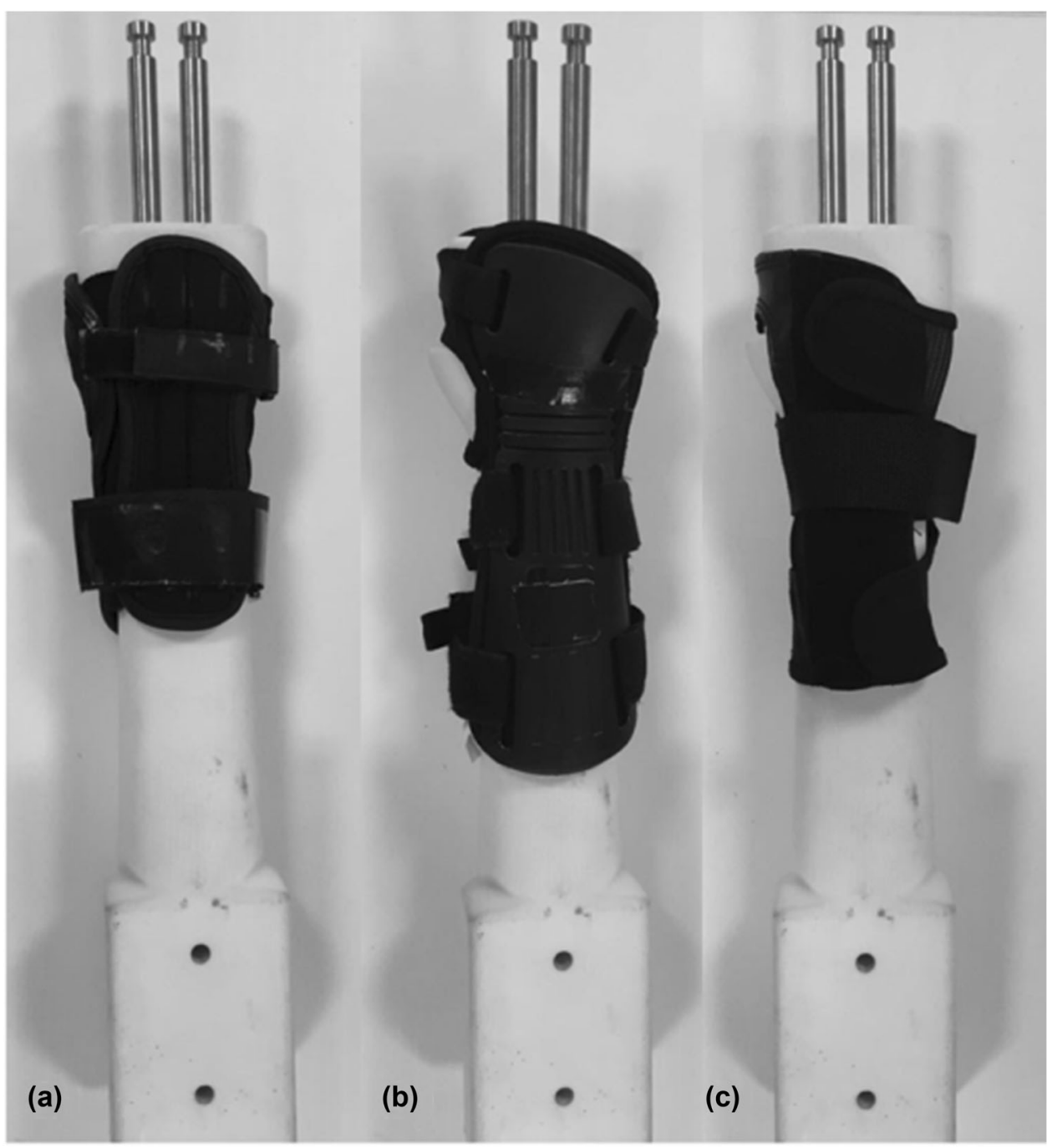

Table 2 Protector measurements

\begin{tabular}{llll}
\hline Protector & $\begin{array}{l}\text { Wrist protector length, } \\
\mathrm{mm}\end{array}$ & $\begin{array}{l}\text { Palmar splint }(\text { width } \times \text { length } \times \text { thickness }), \\
\mathrm{mm}\end{array}$ & $\begin{array}{l}\text { Dorsal splint }(\text { width } \times \text { length } \times \text { thickness }), \\
\mathrm{mm}\end{array}$ \\
\hline $\begin{array}{l}\text { Short } \\
\text { snowboarding }\end{array}$ & 160 & Three splints $(8 \times 70 \times 7)$ & Two splints $(10 \times 145 \times 6)$ \\
$\begin{array}{l}\text { Long } \\
\text { snowboarding }\end{array}$ & 212 & One splint $(70 \times 205 \times 6)$ & One splint $(70 \times 210 \times 10)$ \\
\begin{tabular}{l} 
Roller sports \\
\hline
\end{tabular} & 183 & One splint $(35 \times 155 \times 8)$ & One splint $(30 \times 135 \times 7)$ \\
\hline
\end{tabular}

mounted on the scanned surrogate exceeded the limit of the load cell $(500 \mathrm{~N})$ before the hand could be displaced to $90^{\circ}$, resulting in an extension angle of $84 \pm 0.2^{\circ}$ at the end of the test. In these cases, alternative statistical tests to compare two surrogates rather than three were used like independent $t$ test (if data were normally distributed) or Mann-Whitney test (if data were not normally distributed).

\section{Results}

Significance test results and effect sizes for each pair of surrogates wearing each protector at four angles are presented in Table 3. Statistically significant differences exist in torque between the three surrogates in $78 \%$ of all tested cases. All cases except one demonstrate a moderate to large effect size. In all cases, the geometric and scanned 
Table 3 Inferential statistics, significance test results and effect sizes between surrogates for torque measurements at four different angles

\begin{tabular}{|c|c|c|c|c|c|c|c|}
\hline \multirow[t]{2}{*}{ Protector } & \multirow[t]{2}{*}{ Angle } & \multicolumn{3}{|l|}{$p$} & \multicolumn{3}{|c|}{ Effect size } \\
\hline & & EN-Geo & EN-Scan & Geo-Scan & EN-Geo & EN-Scan & Geo-Scan \\
\hline \multirow[t]{4}{*}{ Roller sports } & 35 & - & $0.202^{\mathrm{e}}$ & - & - & -0.31 & - \\
\hline & 55 & $0^{* \mathrm{c}}$ & $0.029^{c}$ & $0 * \mathrm{c}$ & -0.84 & -0.57 & -0.84 \\
\hline & 80 & $0 *^{\mathrm{c}}$ & $0.021^{\mathrm{c}}$ & $0^{* \mathrm{c}}$ & -0.84 & -0.6 & -0.84 \\
\hline & 90 & $0.097^{\mathrm{b}}$ & $0.019 * \mathrm{~b}$ & $0.003 * \mathrm{~b}$ & 0.51 & -0.76 & -0.8 \\
\hline \multirow[t]{4}{*}{ Short snowboarding } & 35 & $0.003 * \mathrm{~b}$ & $0.547^{\mathrm{b}}$ & $0.032 *^{\mathrm{b}}$ & 0.74 & -0.26 & -0.62 \\
\hline & 55 & $0^{* \mathrm{~b}}$ & $0.161^{\mathrm{b}}$ & $0.009 * \mathrm{~b}$ & 0.83 & -0.46 & -0.73 \\
\hline & 80 & $0.001 * \mathrm{~b}$ & $0.007 * \mathrm{~b}$ & $0.001 *^{\mathrm{b}}$ & 0.81 & -0.73 & -0.83 \\
\hline & 90 & $0.162^{\mathrm{b}}$ & $0^{* \mathrm{~b}}$ & $0^{* \mathrm{~b}}$ & -0.46 & -0.88 & -0.87 \\
\hline \multirow[t]{4}{*}{ Long snowboarding } & 35 & $0.004 *^{\mathrm{a}}$ & $0.006^{*^{\mathrm{a}}}$ & $0^{*^{\mathrm{a}}}$ & 0.77 & -0.61 & -0.86 \\
\hline & 55 & $0^{* \mathrm{c}}$ & $0 * \mathrm{c}$ & $0 * \mathrm{c}$ & -0.84 & -0.84 & -0.84 \\
\hline & 80 & $0.001 *^{\mathrm{c}}$ & $0 *^{\mathrm{c}}$ & $0.001 * \mathrm{c}$ & -0.84 & -0.84 & -0.84 \\
\hline & 90 & $0 * \mathrm{~d}$ & - & - & 0.97 & - & - \\
\hline
\end{tabular}

Statistical tests performed: ${ }^{\mathrm{a}}$ one way ANOVA, ${ }^{\mathrm{b}}$ Welch ANOVA, ${ }^{\mathrm{c}}$ Kruskal-Wallis, ${ }^{\mathrm{d}}$ independent $t$ test, 'Mann-Whitney $U$ test, * indicates a significant difference. Magnitude of effect measured using Pearson's correlation coefficient

EN EN 14120, Geo geometric, Scan scanned surrogates were significantly different $(p<0.005)$ with large effect sizes. EN 14120 and the geometric surrogate were significantly different in $80 \%$ of measured instances; EN 14120 surrogate and the scanned surrogate were significantly different in $55 \%$ of measured instances.

Table 4 presents the coefficient of variation for each protector on each surrogate at the four angles of interest and the mean coefficient of variation for the three surrogate designs. The EN 14120 and geometric surrogate have similar mean coefficients of variation of 24 and $23 \%$, respectively, while the scanned surrogate had a higher mean coefficient of variation of $31 \%$. Figure 4 a shows the first-order polynomial functions and the mean torque for the four angles across eight repeats for one condition. Figure $4 \mathrm{~b}-\mathrm{d}$ shows the torque-angle relationship across all three arms for each protector. In all cases, torque increased with hand extension angle.

Figure 5 shows that ranking order of protector stiffness was generally consistent across surrogates, except for three conditions. In the majority of cases (90\%), the long snowboarding protector exhibited the highest stiffness, requiring a larger torque to reach each hand angle. In contrast, the short snowboarding protector tended to exhibit the lowest stiffness (83\% of cases), with the roller sport protector showing intermediate behaviour. Exceptions include, (1) the EN 14120 surrogate at $35^{\circ}$, (2) the scanned
Table 4 Coefficient of variation (CV) for each protector on each surrogate at the 4 angles of interest

\begin{tabular}{lllll}
\hline Protector & Angle & \multicolumn{2}{l}{ Coefficient of variation (\%) } \\
\cline { 5 - 6 } & & EN 14120 & Geometric & Scanned \\
\hline Roller sports & 35 & 84 & - & 60 \\
& 55 & 45 & 26 & 34 \\
& 80 & 18 & 30 & 28 \\
Short snowboarding & 90 & 12 & 42 & 27 \\
& 35 & 25 & 24 & 47 \\
& 55 & 16 & 16 & 38 \\
Long snowboarding & 80 & 37 & 40 & 44 \\
& 90 & 13 & 9 & 23 \\
& 35 & 23 & 30 & 24 \\
Mean CV for each arm based on all cases (\%) & 55 & 8 & 12 & 15 \\
& 80 & 6 & 8 & 5 \\
\end{tabular}



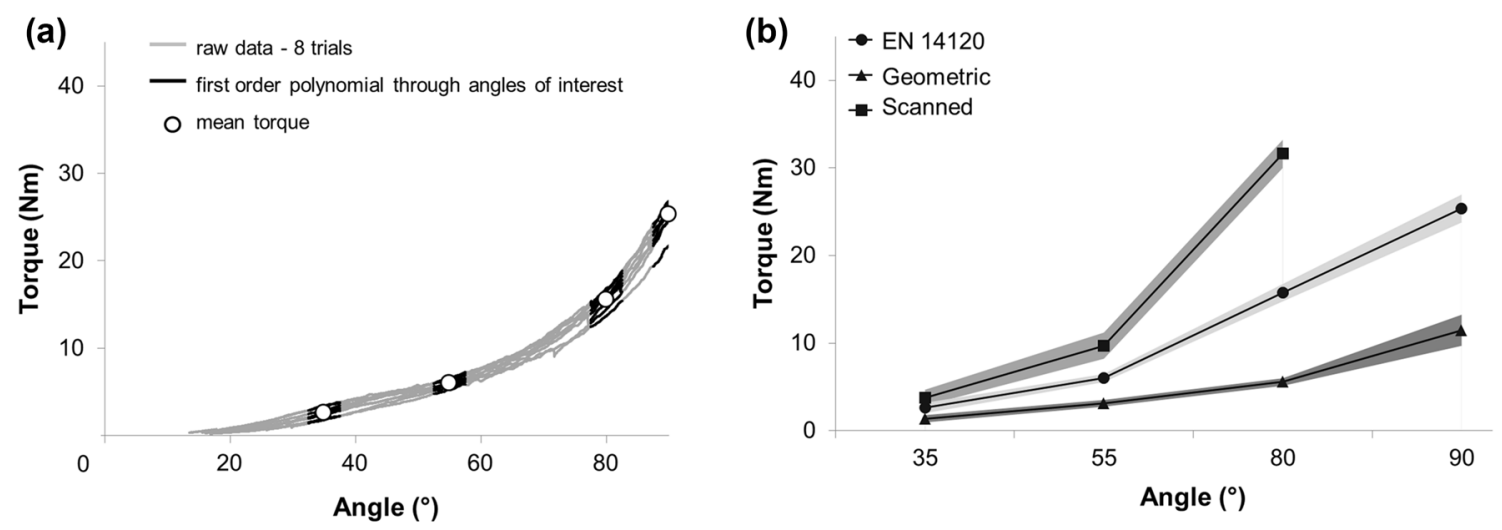

(c)

(d)
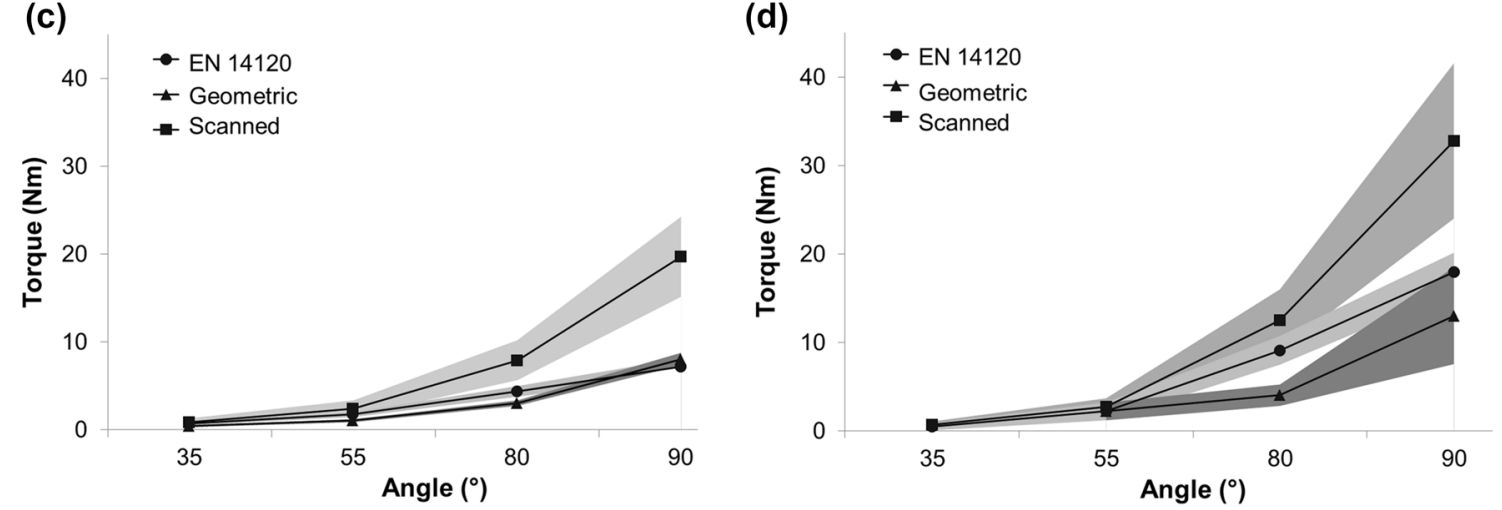

Fig. 4 a Raw data and mean torque at angles of interest for long snowboarding protector on EN:14120 surrogate, surrogate comparison for: b long snowboarding, $\mathbf{c}$ short snowboarding protector, $\mathbf{d}$ roller sports protector

Fig. 5 Comparison of extension torque at each angle for all protectors mounted on all three surrogates

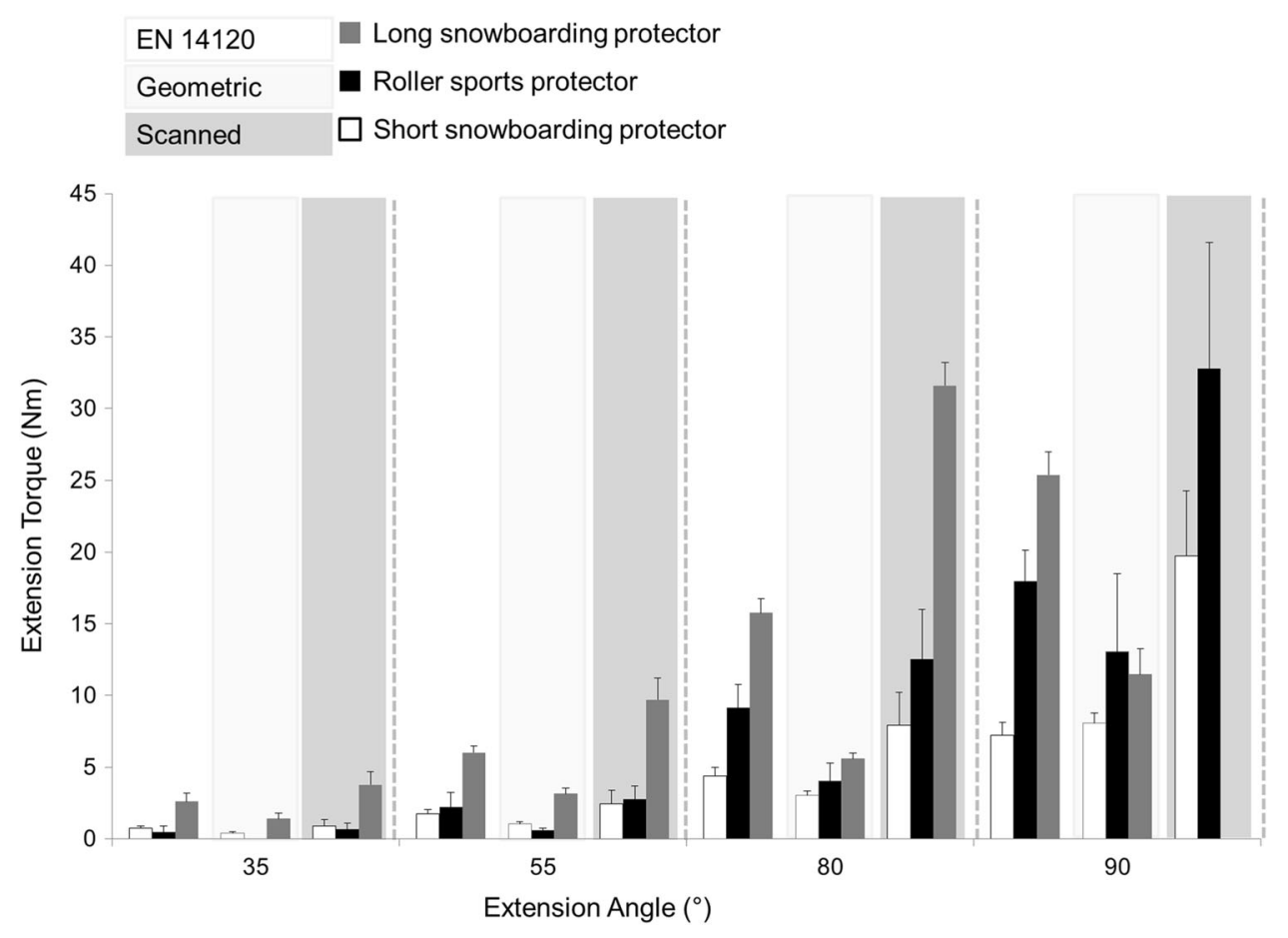

surrogate at $35^{\circ}$, in both cases, the short snowboarding protector required marginally more torque $(0.3 \mathrm{Nm})$ than

the roller sports protector and (3) the geometric surrogate at $90^{\circ}$, where the roller sports protector required slightly more 
torque $(1.6 \mathrm{Nm})$ than the long snowboarding protector. From Fig. 5, it can also be seen that the relative difference in stiffness between protectors changed between surrogates. The smallest differences in protector stiffness were measured when using the geometric surrogate. For example, consider the short and long snowboarding protector at a hand angle $80^{\circ}$. For these two protectors mounted to the EN 14120, the difference in torque was $11.4 \mathrm{Nm}$, a difference of $2.5 \mathrm{Nm}$ when mounted to the geometric surrogate and $23.7 \mathrm{Nm}$ difference for the scanned surrogate.

\section{Discussion}

Surrogate design significantly influences the measured stiffness of snowboarding wrist protectors in a quasi-static bending test. The scanned surrogate required larger torques to displace the hand to each angle (Fig. 4b-d), which may be due to the size of the forearm. The scanned surrogate had the largest volume, with the biggest wrist and forearm circumferences (Table 1), these are the two metrics that have the greatest difference between the selected participant and 50th percentile measures. Given that in reality there is no such thing as an average 50th percentile human, finding a participant with all the equivalent measures is very difficult. Differences in protector performance with surrogate design have implications for the snowboarding wrist protector standard under development. Threshold values in test standards should be linked to surrogates and should not be considered to be transferable across different designs. Whilst the stiffness ranking of the three protectors tended to be consistent across all three surrogates, the relative difference in protector performance varied. The smallest differences in protector stiffness were measured when using the geometric surrogate (Fig. 5). The importance of these relative differences in protector stiffness measurements is likely to be dependent on the application. When determining whether protectors meet the pass criteria for a test in a standard these relative differences are likely to be less of an issue than when undertaking work to determine whether products exhibit different performance, and linking these differences to design parameters for example. Surrogate design is an important consideration when comparing protector stiffness results between laboratories, test houses and research studies.

The coefficient of variation provides insight into the repeatability of the surrogate design. When considering the overall mean coefficient of variation the geometric (23\%) and EN 14120 (24\%) surrogates perform in a similar manner, whilst the scanned surrogate was more variable (30\%). These results imply that the new geometric surrogate is equivalent to the EN 14120 surrogate in terms of repeatability, while the scanned surrogate is worse. Whilst the scanned surrogate is a more accurate representation of the human arm, it has a more complex discontinuous shape. The increased biofidelic accuracy results in a shape with more surface features and irregularities, as details such as muscles and bones are captured. Given the rigid nature of the surrogate, these anatomical features are likely to increase the resistance between the protector and surrogate influencing the fit, resulting in greater variation between trials. The inclusion of a more pronounced thumb in the two new surrogates results in a visibly better fit between the surrogate and the protector, but does not appear to improve repeatability based on the results presented here.

Both the geometric and scanned surrogates are improvements on the current gold standard EN 14120 surrogate, because their geometry better represents a human hand and wrist, they facilitate testing of protectors integrated into gloves and are based on published anthropometric data. The scanned surrogate required participant recruitment to develop and is not easily communicated via an engineering drawing, thereby limiting its reproducibility. The main limitation of the scanned surrogate is the challenge of identifying participants with the desired wrist and hand size. In contrast, the geometric surrogate provided repeatable measurements; is based on readily available anthropometric data; can be communicated in an engineering drawing; can be scaled and updated as required with relative ease. Therefore, the geometric surrogate approach should lend itself well to test protocols in international standards.

Whilst evidence suggests that the geometric surrogate may be best of the three designs, further work could be done to improve repeatability, such as modifying the surface to incorporate a thin layer of compliant material, as a basic representation of skin, to enhance protector fit and limit unwanted movement. The use of a potentiometer to measure extension angle rather than manual inclinometer measurements would simplify the test protocol. Further testing with a greater number of products could be beneficial to better quantify surrogate repeatability and further our understanding of differences in stiffness between protectors. A limitation of the test method presented here is the quasi-static application of load; whilst this facilitates an understanding of product stiffness related to hyperextension, it does not enable a full assessment of the product protective capacity. A complementary approach employing a dynamic test in which other protective parameters can be measured including: energy absorption and load transfer will be developed.

\section{Conclusion}

This paper presents an investigation into the influence of surrogate design on the stiffness of snowboarding wrist protectors. A well-designed surrogate should: be based on 
relevant dimensions; enable the testing of both stand-alone protectors and those integrated into gloves; detect differences between protectors in a repeatable manner. This study has shown that the design of the surrogate significantly influences the measured stiffness of wrist protectors. International standards must link pass thresholds to specific surrogate designs. It is recommended that the geometric surrogate design is adopted as the standard for product testing when quasi-statically testing snowboard wrist guards.

Open Access This article is distributed under the terms of the Creative Commons Attribution 4.0 International License (http://creative commons.org/licenses/by/4.0/), which permits unrestricted use, distribution, and reproduction in any medium, provided you give appropriate credit to the original author(s) and the source, provide a link to the Creative Commons license, and indicate if changes were made.

\section{References}

1. Michel FI, Schmitt K-U, Greenwald RM, Russell K, Simpson FI, Schulz D et al (2013) White paper: functionality and efficacy of wrist protectors in snowboarding - towards a harmonized international standard. Sport Eng 16:197-210

2. Hagel BE, Goulet C, Platt RW, Pless IB (2004) Injuries among skiers and snowboarders in Quebec. Epidemiology 15(3):279-286

3. Kim S, Endres NK, Johnson RJ, Ettlinger CF, Shealy JE (2012) Snowboarding injuries: trends over time and comparisons with alpine skiing injuries. Am J Sports Med 40(4):770-776

4. Bianchi G, Brügger O, Niemann S (2017) Skiing and snowboarding in Switzerland: trends in injury and fatality rates over time. In: Scher IS, Greenwald RM, Petrone N (eds) Snow sports trauma and safety: conference proceedings of the international society for skiing safety: 21st volume. Springer International Publishing, Cham, pp 29-39

5. Michaud P-A, Renaud A, Narring F (2001) Sports activities related to injuries? A survey among 9-19 year olds in Switzerland. Inj Prev J Int Soc Child Adolesc Inj Prev 7:41-45

6. Sasaki K, Takagi M, Ida H, Yamakawa M, Ogino T (1999) Severity of upper limb injuries in snowboarding. Arch Orthop Trauma Surg 119:292-295

7. Ekeland A, Rødven A, Heir S (2017) Injury trends in recreational skiers and boarders in the 16-year period 1996-2012. In: Scher IS, Greenwald RM, Petrone N (eds) Snow sports trauma and safety: conference proceedings of the international society for skiing safety: 21st volume. Springer International Publishing, Cham, pp 3-16

8. Costa-Scorse BA, Hopkins WG, Cronin J, Bressel E (2017) New Zealand snow sports injury trends over five winter seasons 2010-2014. In: Scher IS, Greenwald RM, Petrone N (eds) Snow sports trauma and safety: conference proceedings of the international society for skiing safety: 21 st volume. Springer International Publishing, Cham, pp 17-28

9. Russell K, Hagel B, Francescutti LH (2007) The effect of wrist guards on wrist and arm injuries among snowboarders: a systematic review. Clin J Sport Med 17(2):145-150

10. Whiting W, Zernicke R (2008) Biomechanics of musculo-skeletal injury, 2nd edn. Human Kinetics Publishers, Champaign

11. Bartlett R, Bussey M (2013) Sports biomechanics: reducing injury and improving performance. Routledge, London

12. McIntosh A (2012) Biomechanical considerations in the design of equipment to prevent sports injury. J Sport Eng Technol 226(3-4):193-199

13. Levy D, Hubbard M, McNeil JA, Swedberg A (2015) A design rationale for safer terrain park jumps that limit equivalent fall height. Sport Eng 18:227-239

14. McNeil JA, Hubbard M, Swedberg AD (2012) Designing tomorrow's snow park jump. Sport Eng 15:1-20

15. Kuhn EN, Miller JH, Feltman B, Powers AK, Sicking D, Johnston JM (2017) Youth helmet design in sports with repetitive lowand medium-energy impacts: a systematic review. Sport Eng 20:29-40

16. Hwang IK, Kim KJ (2004) Shock-absorbing effects of various padding conditions in improving efficacy of wrist guards. J Sport Sci Med 3(1):23-29

17. Schmitt K-U, Michel F, Staudigl F (2012) Testing damping performance and bending stiffness of snowboarding wrist protectors. J ASTM Int 9(4):104-119

18. International Organization for Standardization (2016) ISO/CD 20320 Protective clothing for use in snowboarding-wrist protectors-requirements and test methods. https://www.iso.org/ standard/67665.html. Accessed 23 June 2017

19. Adams C, James D, Senior T, Allen T, Hamilton N (2016) Development of a method for measuring quasi-static stiffness of snowboard wrist protectors. Procedia Eng 147:378-383

20. Tilley AR, Henry Dreyfuss Associates (2001) The measure of man and woman: human factors in design. Whitney Library of Design, New York

21. Peebles L, Norris B (1998) Adultdata: the handbook of adult anthropometric and strength measurements: data for design safety. Department of Trade and Industry, Nottingham

22. Pheasant S (2001) Bodyspace: anthropometry, ergonomics and the design of work, 2nd edn. Taylor \& Francis, London

23. European Committee for Standardization (2003) BS EN 420:2003+A1:2009 protective gloves-general requirements and test methods. https://shop.bsigroup.com/ProductDetail/?pid= 000000000030164591. Accessed 5 Mar 2018

24. Materialise. (2017) Laser sintering material properties. http:// www.materialise.com/en/manufacturing/materials/pa-12-sls. Accessed 18 Jan 2018

25. NatureWorksLLC (2018). PLA material information. http://www. faberdashery.co.uk/wp-content/uploads/2011/08/4043D-PLAMaterial-info.pdf. Accessed 18 Jan 2018

26. Greenwald RM, Simpson FH, Michel FI (2013) Wrist biomechanics during snowboard falls. Proc Inst Mech Eng Part P J Sport Eng Technol 227(4):244-254

27. Field A (2009) Discovering statistics using SPSS. SAGE, London

28. Cohen J (1998) Statistical power analysis for the behavioural sciences, 2nd edn. Lawrence Erlbaum Associates, Hillsdale 